\title{
Propagation of Electromagnetic Waves in Terrestrial Environment for Applications in Wireless Telecommunications
}

\author{
Panayiotis Frangos, ${ }^{1}$ Dwight Jaggard, ${ }^{2}$ Seil Sautbekov, ${ }^{3}$ Georgi Georgiev, ${ }^{4}$ and Sava Savov \\ ${ }^{1}$ School of Electrical and Computer Engineering, National Technical University of Athens, 9 Iroon Polytechniou Street, \\ Zografou, 15773 Athens, Greece \\ ${ }^{2}$ Moore School of Electrical Engineering, University of Pennsylvania, Philadelphia, PA, USA \\ ${ }^{3}$ Department of Physics, Eurasian National University, Astana, Kazakhstan \\ ${ }^{4}$ Department of Mathematics and Informatics, University of Veliko Tarnovo, Veliko Tarnovo, Bulgaria \\ ${ }^{5}$ Department of Electrical Engineering, Technical University of Varna, Varna, Bulgaria
}

Correspondence should be addressed to Panayiotis Frangos; pfrangos@central.ntua.gr

Received 11 August 2014; Accepted 11 August 2014; Published 8 September 2014

Copyright (C) 2014 Panayiotis Frangos et al. This is an open access article distributed under the Creative Commons Attribution License, which permits unrestricted use, distribution, and reproduction in any medium, provided the original work is properly cited.

In the time period of about three months, fourteen papers dedicated to this special issue were submitted to me as a guest editor. From them nine were rejected, while five were accepted for publication after minor changes.

Here I would like to make a short characteristic of the five accepted articles. The paper entitled "Calculation of differential propagation constant determined by plant morphology using polarimetric measurement," by C. Hu and $\mathrm{N}$. Li, investigates one important problem related to measurement of polarimetric properties of different materials. In order to validate this phenomenon, the mathematical relation between the differential propagation constant of forest vegetation and its polarized echo is derived by using backscattering power profile. The accurate copolarized data of 3-10 GHz frequency-domain of small trees are obtained by indoor wideband polarimetric measurement system. At low frequencies, the plants with structural features presented oriented distribution. However, the plants show random distribution of the echoes at higher frequencies, which is mainly from the canopy. The research provides important information to choose the coherence models employing the parameters retrieval of vegetations.

The paper entitled "Fast and reliable locating of points with the maximum value of total electromagnetic field using a modern experimental method for radio coverage in urban environment," by P. S. Medjedovic and D. S. Suka, explores one modern experimental method for determination of radio coverage in urban environment. The ultimate goal of the project is the installation of a monitoring network for continuous measurement and recording of nonionizing electromagnetic (EM) radiation levels in the wider area of Banja Luka city centre covering about $10 \mathrm{~km}^{2}$. For this reason it was necessary to make an optimal choice from a dozen of measurement points with the maximum value levels of total EM field. It was a difficult task, because in this case, with the resolution of $5 \mathrm{~m} \times 5 \mathrm{~m}$, approximately 400.000 points should be examined. Therefore, the pedestrian zones, areas, and routes with significantly smaller surfaces but with the largest flow of people have been examined, so the time required for their mapping with values of total EM field has been reduced to a more realistic framework.

The paper "The radiation problem from a vertical Hertzian dipole antenna above flat and lossy ground: novel formulation in the spectral domain with closed-form analytical solution in the high frequency regime," by K. Ioannidi et al., makes a novel analytical treatment of one classical problem: the radiation of a short vertical dipole above the ground. The problem is formulated in a novel spectral domain approach, and by inverse three-dimensional Fourier transformation the expressions for the received electric and magnetic (EM) field in the physical space are derived as one-dimensional integrals over the radial component of wave vector, in 
cylindrical coordinates. This formulation appears to have inherent advantages over the classical formulation by Sommerfeld, performed in the spatial domain, since it avoids the use of the so-called Hertz potential and its subsequent differentiation for the calculation of the received EM field. Subsequent use of the stationary phase method (SPM) in the high frequency regime yields closed-form analytical solutions for the received EM field vectors, which coincide with the corresponding reflected EM field originating from the image point. In this way, we conclude that the so-called in the literature "space wave" (line of sight plus reflected EM field) represents the total solution of the Sommerfeld problem in the high frequency regime, in which case the surface wave can be ignored.

The paper "Determination of measurement points in urban environments for assessment of maximum exposure to EMF associated to a base station," by A. Linhares et al., is dedicated to measurement related to assessment of the maximum exposure to electromagnetic field radiated by the base station. A base station (BS) antenna operates in accordance with the established exposure limits if the values of electromagnetic fields (EMF) measured in points of maximum exposure are below these limits. In the case of BS in open areas, the maximum exposure to EMF probably occurs in the antenna's boresight direction, from a few tens to a few hundred meters away. This is not a typical scenery for urban environments. However, in the line of sight (LOS) situation, the region of maximum exposure can still be analytically estimated with good results.

The paper entitled "Rigorous 2D model for study of pulsed and monochromatic waves propagation near the Earth's surface," by S. S. Sautbekov et al., investigates a rigorous $2 \mathrm{D}$ model for study of transient electromagnetic fields. In the paper, a model is analyzed which allows solving rather complex 2D problems of the electromagnetic wave propagation with a required accuracy using ordinary personal computers. The problems are of great importance for the theory and practical applications. The basis for constructing models of the kind is the exact absorbing conditions whose use makes it possible to reduce the original open initial boundary value problems to equivalent closed problems, that is, to such problems which can be solved numerically using the standard net methods.

\author{
Panayiotis Frangos \\ Dwight Jaggard \\ Seil Sautbekov \\ Georgi Georgiev \\ Sava Savov
}



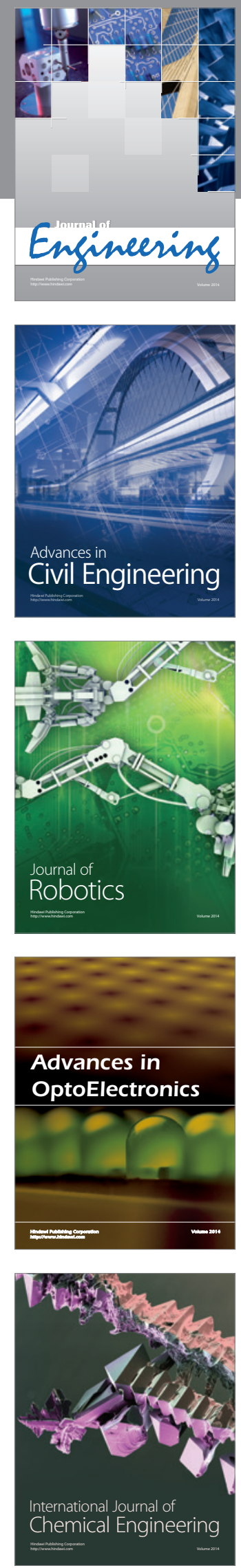

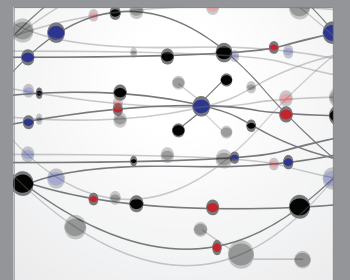

The Scientific World Journal
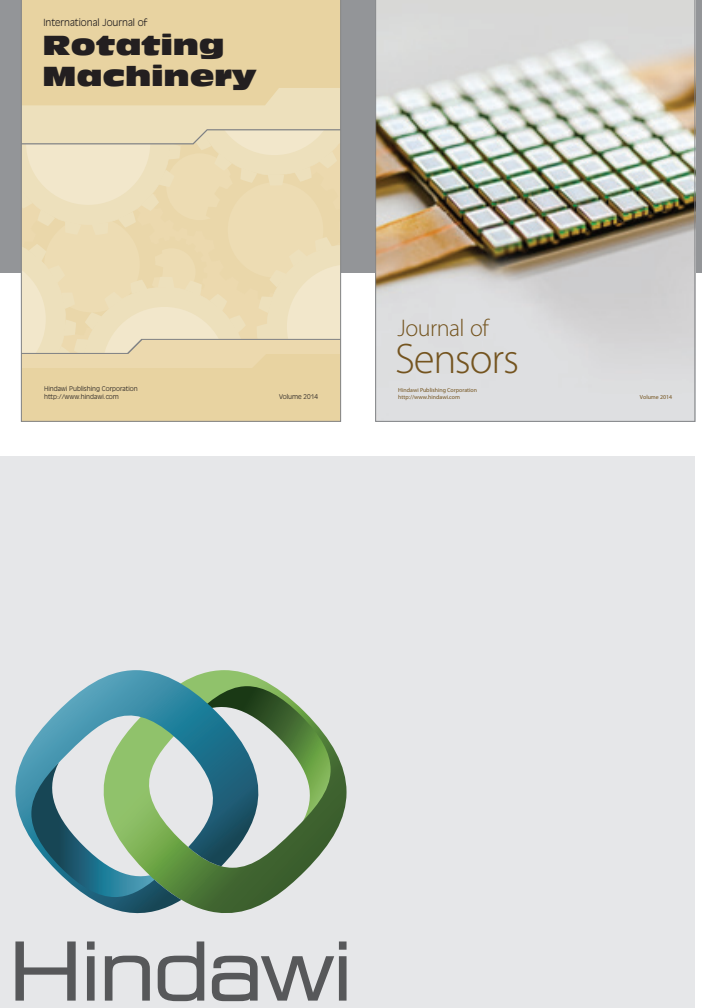

Submit your manuscripts at http://www.hindawi.com
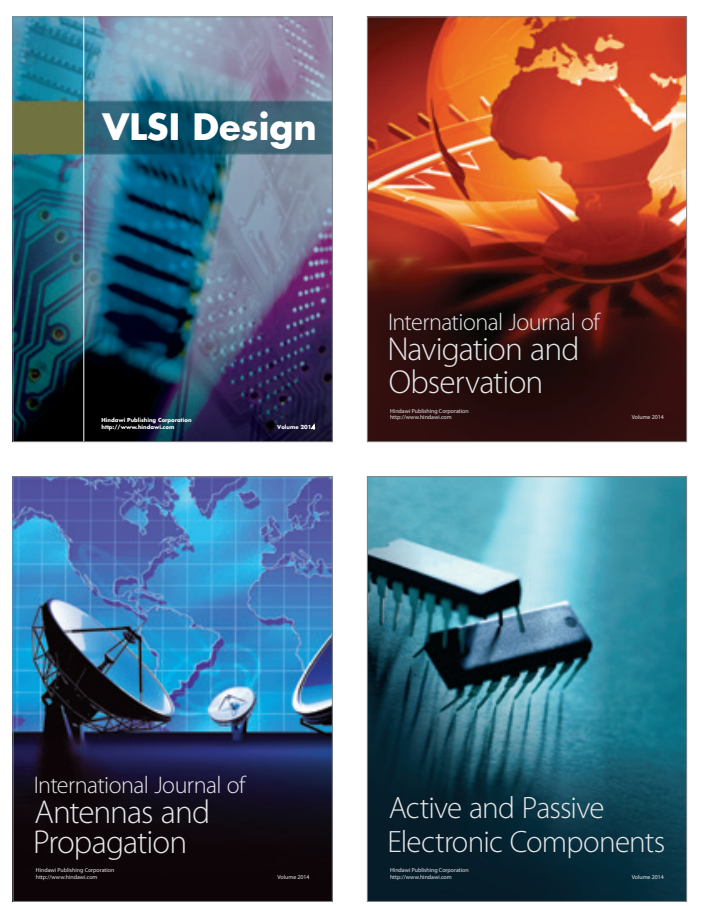
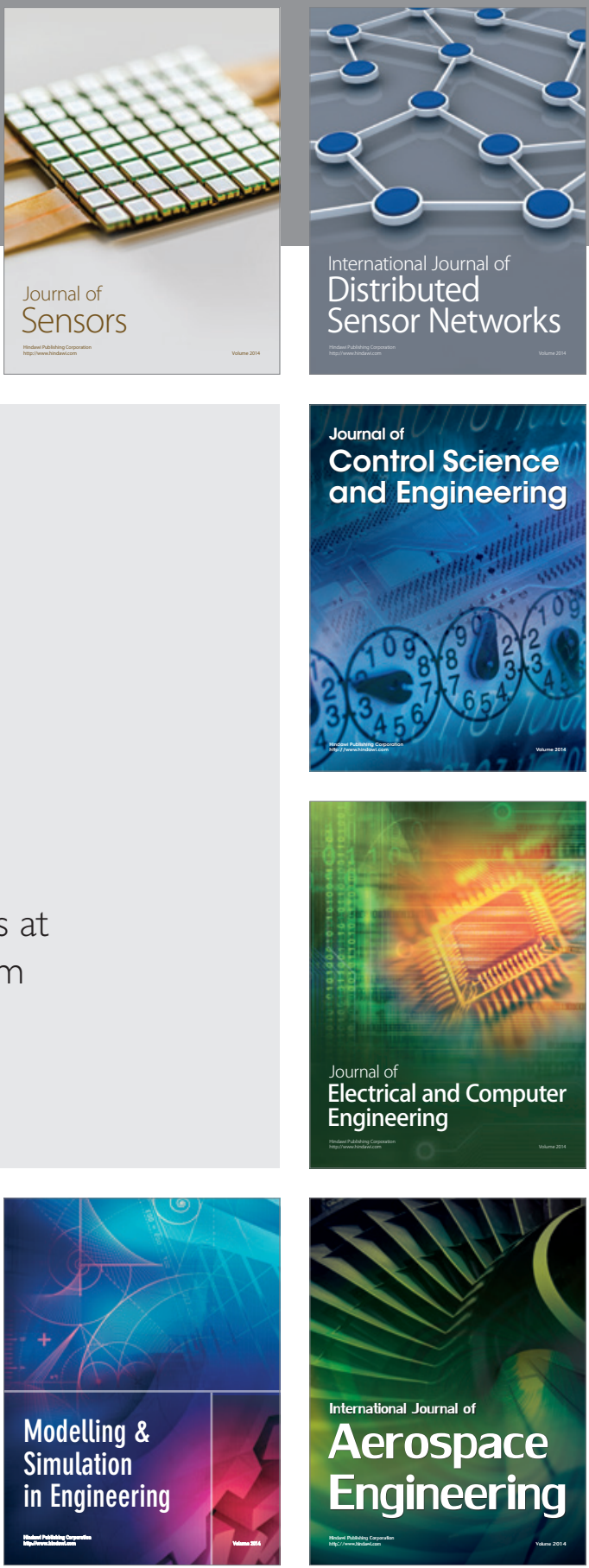

Journal of

Control Science

and Engineering
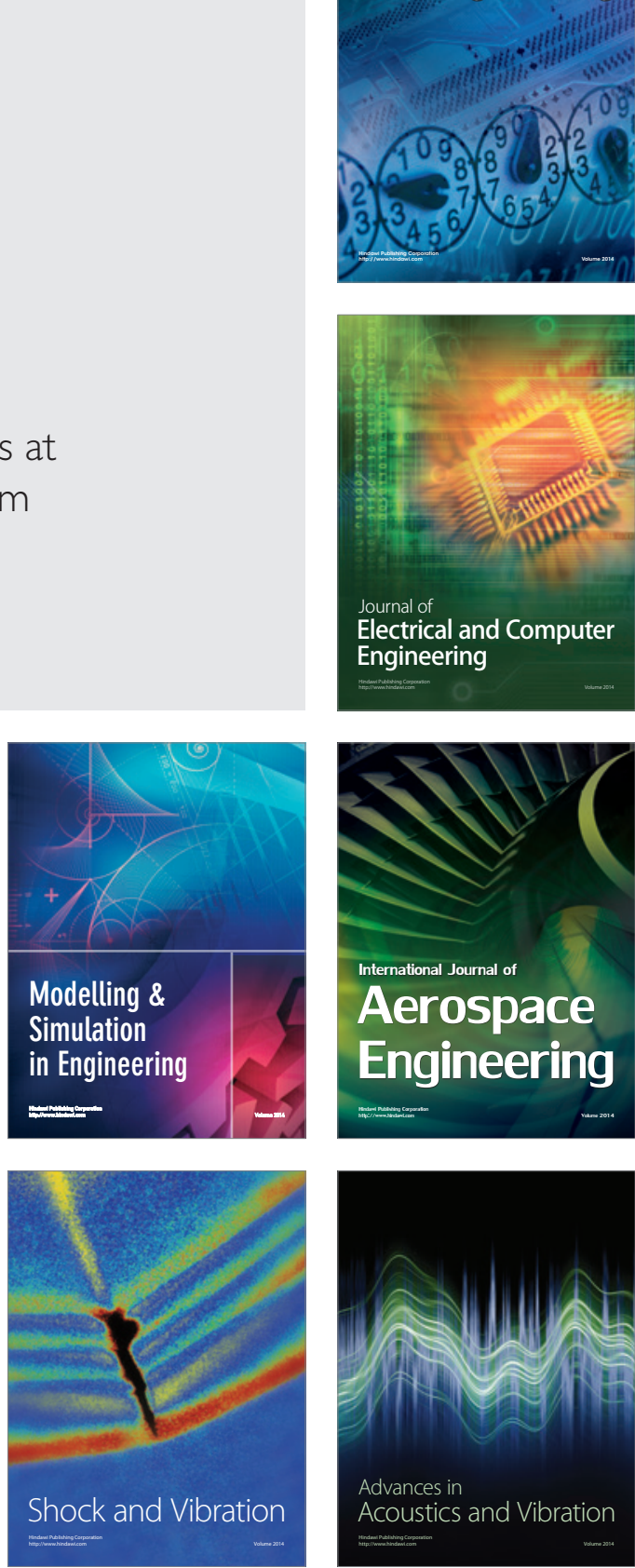